Bundesgesundheitsbl 2020 · 63:1084-1093 https://doi.org/10.1007/s00103-020-03196-7 Online publiziert: 5 . August 2020

(c) Der/die Autor(en) 2020

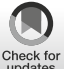

Alf Trojan

Institut für Medizinische Soziologie, Universitätsklinikum Hamburg-Eppendorf, Hamburg, Deutschland

\title{
Integrierte Gesundheitsbericht- erstattung auf Landes- und kommunaler Ebene: Initiativen und Ansätze der letzten 20 Jahre
}

\section{Einleitung}

Für eine möglichst umfassende Gesundheitsberichterstattung (GBE) sollten Kommunen und Länder auf Daten verschiedener Politiksektoren zurückgreifen können, in denen Determinanten der Gesundheitsförderung zu finden sind. Diese Forderung spiegelt sich in dem Begriff ,integrierte Gesundheitsberichterstattung“ wider.

Die Idee, integrierte Gesundheitsberichte $\mathrm{zu}$ verfassen, ohne dass sie so genannt wurden, ist nicht neu: In historischen Rückblicken wird z. B. schon in der Antike der griechische Arzt Hippokrates als Zeuge angerufen [1]. An anderer Stelle wird anhand von nicht weniger als 6 Berichten über Hamburg und Altona (beginnend 1801) aufgezeigt, in welchem Maße den Autoren bewusst war, wie viele Bereiche in den Blick genommen werden müssen, um die Einflussfaktoren auf „Stadt-Gesundheit" abzubilden [2]. Im Gesundheitsbericht Hamburgs von 1901 wird formuliert: „So ist das vorliegende Buch entstanden, ... die Krankengeschichte einer ganzen Stadt zu schreiben und aus ihr den Gewinn zu ziehen, den eine sorgfältige Anamnese am Bett des einzelnen Kranken gewährt“ [3].

Als Ende der 1980er-Jahre in Deutschland das Thema „Gesundheitsberichterstattung“ - nach langer Zeit eines Schattendaseins - gesundheitspolitisch wiederentdeckt wurde, herrschte zwar überwiegend ein begrenztes Konzept relevanter Indikatoren vor. Aber schon im Pilotbericht für die Länderberichterstattung gab es das „Themenfeld 5: Gesundheitsrisiken aus der natürlichen und technischen Umwelt" mit Indikatoren für Klima, Wasser, Boden, Luft, Lärm, Müllentsorgung, Umweltschadstoffe und Ähnliches mehr. Ungeachtet der nicht immer perfekten Bearbeitung dieser Themen in den Länderberichten war dies doch ein Zeichen für integrative Absichten.

Ungefähr gleichzeitig mit dem Entstehen der Länderberichterstattung wurden im Rahmen des Projektes „Healthy Cities“ auf Basis des Ottawa-Konzepts „Intersektorale Politik“ sehr viel ambitioniertere integrative Indikatorensätze für gesunde Städte konzipiert mit $\mathrm{Pa}$ rametern, wie z.B. Ecosystem Sustainability, Community Strength, Sense of Connectedness oder Diverse City Economy [1]. In den 1990er-Jahren wurde der Gedanke integrativer Berichterstattung und integrierter Stadtpolitik gleichsinnig durch Projekte der Lokalen Agenda 21 und der sozialen Stadtentwicklung weiter befeuert. Im Jahr 2001 konnten Osius et al. [4] eine Übersicht über eine große Anzahl verschiedener Indikatorensysteme ,für eine gesunde und nachhaltige Stadtentwicklung “ zusammentragen. Dabei lag der Schwerpunkt zwar auf dem Schnittbereich von Umwelt und Gesundheit. Mit dem Thema „Nachhaltigkeit“ war jedoch auch ein weiter reichendes Feld von Indikatoren angesprochen, ohne dass der Ausdruck integrative Berichterstattung schon eine Rolle spielte.

Mit der Konzeptionierung und Etikettierung von Gesunde-Stadt-, Lokale-Agenda-21- und Soziale-Stadt-Pro- grammen als integrierte Programme wurde jedoch zunehmend häufiger auch von integrativer oder integrierter (Gesundheits-)Berichterstattung gesprochen. Dabei gab und gibt es keinen allgemeinen Konsens über die Definition dieses Begriffs. In diesem Beitrag gehen wir daher von der Selbstdefinition der Berichterstattung als ,integriert" aus und beziehen auch Gesundheitsberichterstattung ein, wenn sie nur einen Teil des Titels ausmacht (Beispiel: „integrierte Gesundheits- und Sozialberichterstattung“). Die wesentlichen Ebenen der Integration sind: Daten/Indikatoren aus anderen Politikbereichen (insbesondere Umwelt/Nachhaltigkeit und Soziales), Kooperation der beteiligten Politikressorts, ressortübergreifende Ziele und Handlungsvorschläge.

Obwohl diese Ausdehnung der Gesundheitsberichterstattung auf möglichst alle Determinanten der Gesundheit als Desiderat allgemein akzeptiert wurde, scheint sie sich in der Praxis nicht durchgesetzt zu haben: „Der Idealfall einer integrierten (gesundheitsbezogenen) Berichterstattung hat zwar gerade im Bereich der als intersektorale Politik verstandenen Gesundheitsförderung viele Befürworterinnen und Befürworter, stößt bei der Umsetzung auf kommunaler Ebene jedoch auf vielfältige Probleme“ [5].

Stimmt dieser Eindruck oder gibt es im deutschsprachigen Bereich explizite Konzepte und eine öffentlich kommunizierte Praxis integrierter GBE? Anlässlich des vorliegenden Themenheftes des Bun- 
desgesundheitsblattes soll dieser Frage im folgenden Beitrag explorativ nachgegangen werden. Nach einem Abschnitt zum methodischen Vorgehen werden die gefundenen Initiativen und Projekte integrierter GBE vorgestellt und diskutiert.

\section{Methodisches Vorgehen}

Angesichts einer Recherche, die nicht nur wissenschaftliche, sondern auch Praxisinitiativen erfassen sollte, wurde eine einfache Google-Recherche ohne zeitliche Einschränkungen durchgeführt, ergänzt durch eine ebensolche Suche bei Google Scholar. Es wurde mit den Begriffen „integrierte Gesundheitsberichterstattung“ und „integrierte Gesundheitsberichte" sowie ergänzend (um Ansätze aus anderen Bereichen $\mathrm{zu}$ identifizieren) mit ,integrierter Berichterstattung " gesucht, begrenzt auf den deutschsprachigen Raum. Nur bei den gefundenen Links mit aussichtsreichen Hinweisen wurde nach dem Schneeballsystem nach weiterem Material gesucht. Bei Anzeichen, dass sich integrierte Berichte in anderen Ämtern als dem Gesundheitsreferat verbargen, wurde die Suche entsprechend ausgeweitet. Für das weiter unten berichtete Projekt mit 10 Mitgliedern des GesundeStädte-Netzwerks wurde auf den Internetseiten der betreffenden Städte nach der aktuellen Situation der Berichterstattung recherchiert und eine Anfrage an das Kompetenzzentrum „Gesundheitsberichterstattung" des Netzwerks gerichtet. Als Ergebnis wurde eine Recherche im Netzwerk angeregt, jedoch noch nicht durchgeführt.

Einbezogen wurden alle Initiativen und Projekte, bei denen mindestens ein als „integriert“ definierter Bericht auffindbar war. Ausgeklammert von der weiteren Behandlung wurden mehrere Links, die sich auf integrierte Berichterstattung in Wirtschaftsunternehmen bezogen.

Die Schlüsselpersonen aller Projekte wurden angeschrieben, um zu erfahren, ob die Berichterstattung auf Dauer weitergehen sollte und ob andere Initiativen integrierter Berichterstattung bekannt waren. Die 6 Antworten (telefonisch, per E-Mail oder beides; von 8 An- gefragten) wurden, soweit relevant, in die Darstellung einbezogen.

\section{Ergebnisse}

Es wurden 8 Projekte (zu denen es meist mehrere Quellen gab) identifiziert, die überwiegend wissenschaftsinduziert waren bzw. in enger Kooperation zwischen Ämtern und Hochschulen entstanden. 4 Projekte/Initiativen repräsentieren kommunale bzw. städtische integrierte Berichterstattung; in Nordrhein-Westfalen (NRW) gibt es 2 Initiativen von Landesbehörden, die sich aber auf die kommunale Berichterstattung beziehen; in Berlin und im Burgenland/Österreich geht es um integrierte Berichterstattung auf der Landesebene.

\section{Hamburger Initiativen für nachhaltige und gesunde Stadtentwicklung (mit Beteiligung von 10 ostdeutschen Städten)}

In einem ersten Projekt wurde als Teil des Hamburger Verbundes zur „Nachhaltigen Entwicklung in Metropolregionen“ eine Bestandsaufnahme von Leitbildern und Indikatorensystemen durchgeführt, die Ende 2000 in einem Workshop mit relevanten Hamburger Akteuren und Input aus dem damaligen Landesinstitut für den Öffentlichen Gesundheitsdienst (LÖGD) in NRW (Rainer Fehr) diskutiert wurde [6].

Das abschließende Bilanzpapier dieses Projekts [4] floss unmittelbar in ein zweites Projekt ein, das als Praxis-Forschungs-Kooperation konzipiert war und den Titel trug „Gesundheit als integrierendes Leitziel in der Konzeption und Erprobung eines regionalen Berichtssystems nachhaltiger Entwicklung“. Die Gesundheitsämter von 10 ostdeutschen Mitgliedern des GesundeStädte-Netzwerks (2 Berliner Bezirke, Chemnitz, Dresden, Erfurt, Greifswald, Halle, Magdeburg, Rostock und Stralsund) waren bereit, zusammen mit dem Forschungsprojekt einen integrierten Gesundheitsbericht zu erstellen, davon 5 mit Schwerpunkt Kinder- und Jugendliche. Dazu war aus 17 vorgefundenen mehr oder weniger integrierten Indikatorensystemen ein Indikatorensatz entwickelt worden, der sich auf die 7 Bereiche Demografie, kommunale Ökonomie, Gesundheit, Soziales, Bildung, Umwelt sowie Wahlen/Partizipation bezog. Die Quellen, der Auswahlprozess und die Profile der 35 Kernindikatoren für eine „integrierte Basis-Berichterstattung für gesündere Städte und Kommunen“ wurden 2004 zusammen mit dem LÖGD in dessen wissenschaftlicher Reihe publiziert [7].

Als Mindestanforderungen an Integration wurden definiert: die Integration aufDaten- und auf Akteursebene bzw. die Integration auf der methodisch-inhaltlichen Ebene und auf der Ebene der intersektoralen Kooperation im Sinne ressortübergreifender Zusammenarbeit. Als wünschenswert wurden aber zahlreiche weitere integrative Elemente genannt: Integration der Öffentlichkeit (Bürgerbeteiligung), der späteren Nutzerinnen und Nutzer, Integration in bestehende Berichts- und Planungsinstrumente sowie in Sonderprogramme zur Verbesserung der Lebensqualität (gesunde, soziale, zukunftsfähige Stadt u. Ä.; [8]).

Der Prozess der Umsetzung erwies sich als schwierig. Immerhin gab es trotz der heterogenen Ausgangslage für eine integrierte nachhaltigkeitsorientierte kommunale (Gesundheits-)Berichterstattung in jeder Verbundstadt Anknüpfungspunkte für eine Weiterentwicklung der Berichterstattung hin zu einem qualitativ höher $\mathrm{zu}$ bewertenden Maß von Integration, und zwar hinsichtlich der Ziele:

- Integration weiterer Politikfelder in einen bereichsspezifisch verantworteten Bericht,

- stärkere Integration auf der Datenebene (z.B. Verknüpfung der Daten aus dem Gesundheitsbereich mit sozialen Daten im Rahmen der Auswertung von Schuleingangsuntersuchungen) oder die Betonung der sozialen Dimension durch eine sozialräumlich differenzierende Aufbereitung von Daten,

- stärkere Betonung von Handlungsorientierung im Sinne integrierter (d.h. einzelne Ressortgrenzen überschreitender) Empfehlungen, Schlussfolgerungen oder Zielformulierungen für „Taten“, 
Bundesgesundheitsbl 2020 -63:1084-1093 https://doi.org/10.1007/s00103-020-03196-7

(c) Der/die Autor(en) 2020

\section{A. Trojan}

\section{Integrierte Gesundheitsberichterstattung auf Landes- und kommunaler Ebene: Initiativen und Ansätze der letzten 20 Jahre}

\section{Zusammenfassung}

Die Ottawa-Charta von 1986 schuf ein neues Paradigma der Gesundheitsförderung, in dem Konzepte wie intersektorale Politik, Determinanten der Gesundheit und Vermitteln und Vernetzen eine wichtige Rolle spielen. In diesem Rahmen entstand die Forderung, dass Gesundheitsberichterstattung (GBE) in einer „integrierten“ Form zu erbringen sei, also Daten aus ganz verschiedenen Politiksektoren enthalten sollte, in denen Determinanten der Gesundheit zu finden sind. In diesem Beitrag soll der Frage nachgegangen werden, ob es im deutschsprachigen Raum auf kommunaler und Landesebene explizite Konzepte und eine öffentlich kommunizierte Praxis integrierter GBE gibt. Dazu wurde eine explorative Internetrecherche durchgeführt, ergänzt um
E-Mail-Anfragen bei Schlüsselpersonen der Projekte.

Es wurden 8 Projekte identifiziert: 4 davon repräsentieren kommunale bzw. städtische integrierte Berichterstattung; in Nordrhein-Westfalen gibt es 2 Initiativen von Landesbehörden, die sich auf die kommunale Berichterstattung beziehen; in Berlin und im Burgenland (Österreich) geht es um integrierte Berichterstattung auf der Bundeslandebene. Die Projekte werden in stark komprimierter Form vorgestellt. Hauptlimitationen der explorativen Recherche sind die Beschränkung auf explizit als "integriert" bezeichnete Berichterstattung und die eng eingeschränkten Suchbegriffe. Ein positiver Befund ist, dass es einige gute Indikatorenkonzepte, gesetzliche Rahmen- bedingungen und förderliche Faktoren, wie z. B. wissenschaftliche Unterstützung, gibt. An der konkreten Umsetzung integrierter GBE mangelt es aber noch. Mögliche Hürden sind dabei die Komplexität der Programme, fehlende finanzielle, personelle und fachliche Ressourcen, organisatorische und methodische Probleme sowie Ängste und Vorurteile. In verschiedenen Beispielen konnten diese aber überwunden werden. Der Leitfaden Gute Praxis Gesundheitsberichterstattung stützt - explizit, aber etwas zu versteckt - die Forderung nach integrierter Berichterstattung.

Schlüsselwörter Gesundheitspolitik · Intersektorale Politik - Determinanten der Gesundheit . Gesundheitsförderung · Public Health

\section{Integrated health reporting at the communal and federal state level-policy initiatives and approaches of the last 20 years}

\section{Abstract}

The Ottawa Charter produced a new paradigm of health promotion, putting forward concepts such as intersectoral policies, determinants of health, and mediating. In this framework, the demand was made that health reports should be conceptualized as integrative, containing data from all sectors where health determinants can be found. The research question is: Can we identify explicit concepts and a publicly communicated practice of integrated health reporting in the Germanspeaking countries? In order to answer this question, a web search was carried out and supplemented by emailed inquiries to key project personnel.
Eight projects were identified: four projects/initiatives represented local or regional integrated health reporting; in North RhineWestphalia there were two initiatives started by the federal state but relating to local reporting; in Berlin (Germany) and Burgenland (Austria) we found reports for the federal states. The projects are presented in compressed form. Limitations of this explorative research are its restriction to reports explicitly labeled as "integrative" and the narrowly defined search terms. Positive findings were good indicator concepts, some legal frameworks, and other promotive factors, such as scholarly support. However, there are shortcomings in putting integrative reporting into practice. Barriers might be the complexity of programs, missing financial, personal, and professional resources, organizational and methodical problems, as well as anxieties and prejudices. Some examples show that the barriers can be overcome. The guideline "Good Practice Health Reporting" supports the call for integrated reporting explicitly, however too much is hidden in the document.

\section{Keywords}

Health policy · Intersectoral policies . Determinants of health $\cdot$ Health promotion . Public health
- Integration auf der Akteursebene, insbesondere die Stärkung oder Neubildung intersektoraler Kooperationsstrukturen für integrierte Berichterstellung.

Als hilfreich für die Wissenschaftler (aber auch als bedeutsam in der späteren praktischen und wissenschaftlichen Rezeption des Projekts) hat sich ein Stufenmodell erwiesen, das ausgehend von einem monothematischen Standardgesundheitsbericht in 8 aufeinanderfolgenden Stufen intensiverer Integration als oberste Stufe ein Idealmodell integrierter Berichterstattung abbildet ([9]; - Abb. 1).

Da bei Projektende noch nicht alle Berichte der Städte fertig waren, gibt es keinen systematischen Vergleich, wie weit die Städte am Ende gekommen waren. Es gab aber einen abschließenden
Workshop, bei dem die Erfahrungen bzgl Chancen und Hürden ausführlich festgehalten wurden [9] Hierauf wird in der Diskussion im Kontext andernorts gemachter Erfahrungen eingegangen.

Die Nachrecherche auf den Seiten der 10 Gesundheitsämter ergab, dass in 6 Fällen keinerlei Berichterstattung stattfand; in 2 Fällen war sie extrem reduziert und mehrere Jahre zurückliegend und in 2 Fällen fanden sich integrierte Berichte: 


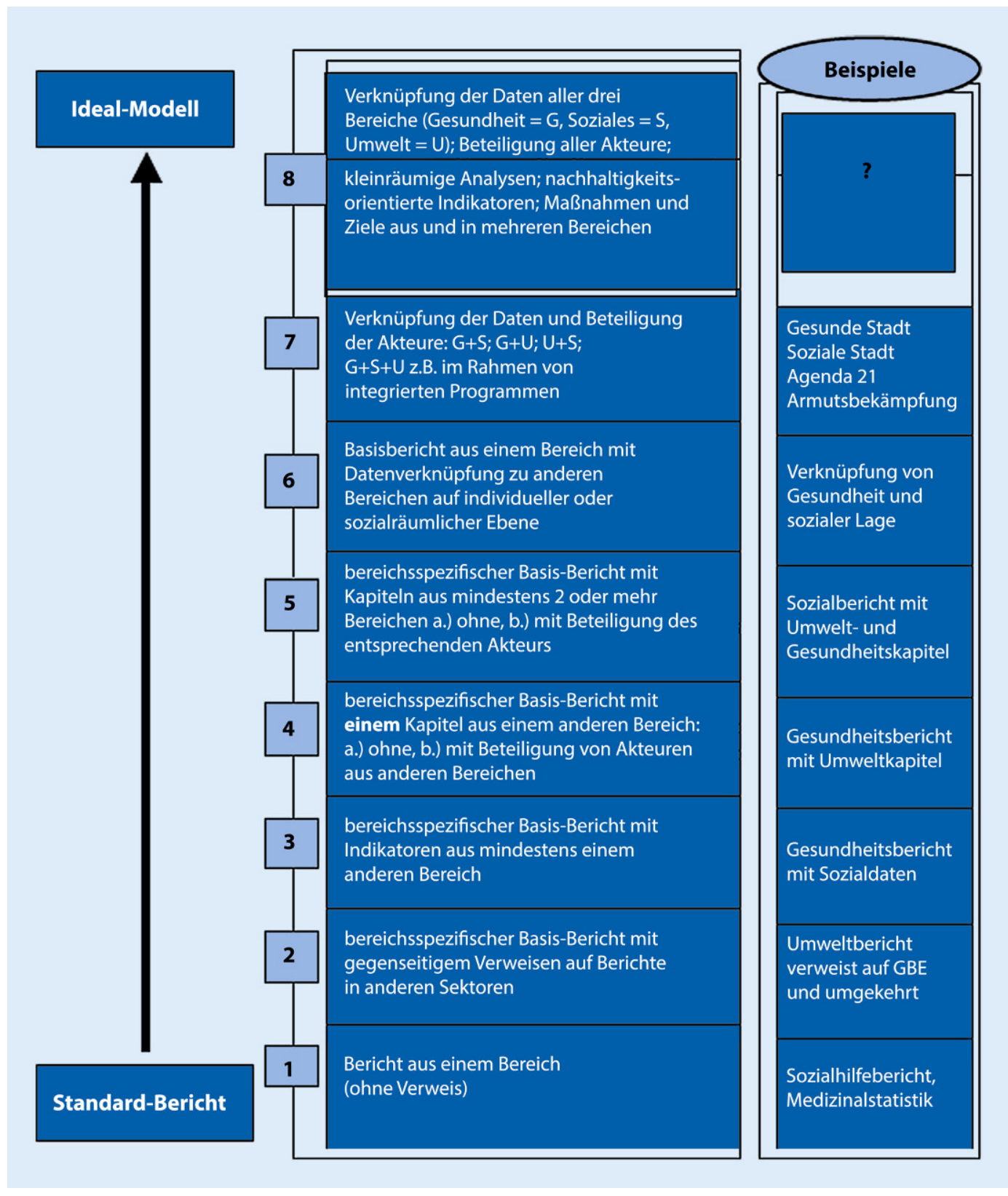

Abb. $1<$ Während man bei den ersten Stufen eigentlich noch nicht von einem „integrierten Bericht" sprechen kann, wird mit Stufe 4 ein gewisses Qualitätsniveau erreicht, das den Begriff rechtfertigt. Die Stufenleiter sollte als Impulsgeber und (Zwischen-)Bilanzhilfe für die verschiedenen Akteure in den jeweiligen Ämtern dienen und für einen Prozess hin zu stärkerer Integration motivieren. (aus [9, S. 76], mit freundlicher Genehmigung des $\odot$ Shaker Verlags)

in Halle unter der Überschrift „Integriertes Stadtentwicklungskonzept" (über das wegen seines minimalen Gesundheitsanteils nicht weiter berichtet wird; [10]) und in Dresden betitelt als „Stadtgesundheitsprofil“" (mit deutlichem Gesundheitsanteil, s. folgenden Abschnitt).

\section{Städtische Berichte: Gera, Weimar, Dresden}

Gera - 2014 hat die Stadt Gera erstmals im Rahmen einer integrierten Sozialberichterstattung Daten und Fakten zum sozialen Leben mit einem lebensweltbezogenen, integrierten Ansatz dargestellt, wobei auch Gesundheit $\mathrm{zu}$ den als wesentlich eingeschätzten Lebensbereichen gehörte. Ziel war die Entwicklung von fachübergreifenden Schlussfolgerungen und kommunalpolitischen Empfehlungen für das Integrierte Stadtentwicklungskonzept der Stadt Gera (ISEK Gera 2030), beschlossen im Mai 2014 durch den Stadtrat [11]. Das Konzept „SOZIALPLAN 2018-2023 Integriertes Handlungskonzept der Stadt Gera" erschien 2018, enthält zahlreiche Gesundheitsaspekte und wurde teilweise durch den Europäischen Sozialfonds er- möglicht [12]. Schon auf Basis des ISEK Gera 2030 wurde eine Masterarbeit aus Sicht der Gesundheitswissenschaften angefertigt für den Fachdienst Gesundheit zur Weiterentwicklung kommunaler Gesundheitsförderung im Rahmen des ISEK Gera 2030 und mit besonderem Schwerpunkt Kinder und Jugendliche [13]. In engem Zusammenhang damit entstand auch Geras „Netzwerk gesunde Kommune“ [14]. Gera ist aktives Mitglied des Gesunde-Städte-Netzwerks.

Die Amtsleiterin des Ressorts Gesundheit schreibt auf Anfrage, dass es wegen mangelnder technischer und per- 
soneller Ressourcen nicht möglich sei, Gesundheitsberichte $\mathrm{zu}$ verfassen, dass Gesundheit aber nachhaltig in der integrierten Sozialplanung vertreten sei [15].

Weimar - Aufbauend auf einem Bericht überSchuleingangsuntersuchungen von 2014 entstand 2017 die „Integrierte Gesundheitsberichterstattung der Stadt Weimar im Kontext der kommunalen Präventionskette" - ebenfalls (wie die Masterarbeit in Gera) sehr stark fokussiert auf (Armuts-)Risiken von Kindern und Jugendlichen [16]. Auf den Internetseiten der Stadt zu Gesundheitsförderung und Prävention wird eine gute Zusammenarbeit beim Aufbau der kommunalen Präventionskette „miteinander! wachsen!“ hervorgehoben und versprochen, dass dem Bericht über Schuleingangsuntersuchungen von 2014 weitere folgen sollen.

Möglicherweise hat für die Initiativen in Gera und Weimar eine Tagung des Thüringer Ministeriums für Soziales, Familie und Gesundheit (Stabsstelle Strategische Sozialplanung) eine Rolle gespielt, die im September 2013 stattfand und den Titel trug: „Integrierte Berichterstattung - Wege und Perspektiven zur Stärkung von Kommunen“" [17].

Dresden - In Dresden wurden seit 1991 insgesamt 5 Ausgaben des sogenannten Stadtgesundheitsprofils im Rahmen des Dresdner WHO-Projektes publiziert [18].

Die bisher letzte von 2016 behandelt die Themenbereiche Demografie, Gesundheitszustand und -versorgung, soziale Lage, Umwelt sowie 3 lebensphasenbezogene Kapitel.

Der Vorlage eines Stadtgesundheitsprofils folgt in der Regel eine Gesundheitskonferenz mit politischen Vertretern, Fachleuten, Vertretern der Sächsischen Landesärztekammer sowie der Krankenkassen, um konkrete Maßnahmen zur weiteren Förderung der Gesundheit der Dresdnerinnen und Dresdner $\mathrm{zu}$ erarbeiten. Dresden engagiert sich seit knapp 30 Jahren im internationalen und deutschen Gesunde-StädteNetzwerk. Wenn es im Vorwort zum Bericht 2016 [18] heißt, dass Gesundheit eine „Aufgabe der gesamten Stadtverwaltung darstellt", zeigt dies, dass hier ein Idealmodell in mehrfacher Hinsicht verwirklicht wird (Orientierung am Health Policy Action Cycle; gesundheitsfördernde Gesamtpolitik bzw. Gesundheit in allen Politikbereichen; große Kontinuität in der integrierten Berichterstattung).

\section{Integrierte kommunale} Berichterstattung NordrheinWestfalen

Schon lange weit entwickelt und auch evaluiert ist die Gesundheitsberichterstattung in NRW. Sie ist eine durch das Gesetz über den Öffentlichen Gesundheitsdienst (ÖGDG) festgeschriebene Aufgabe. Im Rahmen der Evaluation dieses Gesetzes [19] wurde schon 2003 berichtet, dass nur 11 von 52 Kommunen im Erhebungszeitraum keinerlei Gesundheitsberichte verfasst hatten.

2015 wurde unter Nutzung der Datenbank mit kommunalen Gesundheitsberichten [20, 21] von Borrmann und Rosenkötter ermittelt, wie viele Berichte als „integriert“ anzusehen sind [22]. Für die Klassifizierung des Entwicklungsstandes wurde die Stufenleiter genutzt (• Abb. 1). $54(=44 \%)$ der seinerzeit 123 eingestellten Berichte (15 Basisberichte; 39 Spezialberichte) erfüllten mindestens die Kriterien der Stufe 4. Basisberichte konnten mehrheitlich der Stufe $4(n=4)$ oder $5(n=5)$ zugeordnet werden. Von den 39 Spezialberichten entsprachen $15 \mathrm{Be}$ richte in Teilen der Stufe 6 und 3 Berichte der Stufe 7, welche durch Datenverknüpfung und intersektoralen Austausch erreicht wird. Die Autorinnen folgern, dass in NRW Daten aus anderen Politikbereichen relativ häufig von der GBE genutzt werden. Eine umfangreiche Integration, bei der die Akteure kooperieren und auch gemeinsam Handlungsempfehlungen definieren, scheine aber die Ausnahme zu sein.

Seit einiger Zeit werden die Berichte in der Datenbank Kommunale GBE auf Basis der Stufenleiter mit einem „I“ für integrierte Berichterstattung etikettiert, wenn sie die Integrationsstufe 4 oder höher erreicht haben. Von den insgesamt 225 Berichten (Stand: 18.02.2020) waren 123 (37 Basis- und 86 Spezialberichte) entsprechend $55 \%$ als integriert einge- stuft, bei den Spezialberichten überwog das Thema „Kinder und Jugendliche“ mit 52 Berichten (eigene Auszählung).

Neben der GBE ist auch der Masterplan Umwelt und Gesundheit [23] aus dem Ministerium für Umwelt, Landwirtschaft, Natur- und Verbraucherschutz relevant. „Integrierte Berichterstattung: Umwelt, Gesundheit und soziale Lage“ heißt eines der 7 Handlungsfelder des Masterplans, der auch in Zukunft in Kooperation und Austausch mit der Gesundheitsberichterstattung wie auch der Sozialberichterstattung weiter umgesetzt werden soll. Ein Pilotprojekt mit 3 Kommunen hat 2018 zunächst zu einer Tagung geführt, Titel: „Umwelt - Gesundheit - Soziales, - aller guten Dinge sind 3?! Gute Beispiele integrierter kommunaler Berichterstattung“, die im Rahmen der seit vielen Jahren stattfindenden Veranstaltungsreihe „Innovative Ansätze in der kommunalen Sozialberichterstattung " des Sozialministeriums NRW stattfand und gemeinsam von den Ressorts Umwelt, Gesundheit und Soziales (zu dem Thema „integrierte Berichterstattung“) durchgeführt wurde.

Aus dem Austausch dort sind wiederum Handlungsempfehlungen unter der Überschrift „Integrierte Berichterstattung und Planung - Erfolgsfaktoren, Hürden und gute Argumente" hervorgegangen [24]. Die Empfehlungen werden über fachliche Netzwerke in NRW gestreut. Für deren breitere Rezeption und Umsetzung in den Kommunen muss allerdings mit Zeiträumen von 3-5 Jahren gerechnet werden.

Auch aus Gesprächen mit den 2 Schlüsselpersonen in NRW ging deutlich hervor, dass der Implementierung integrierter kommunaler Berichterstattung seitens der relevanten Landesbehörden ein hoher Stellenwert beigemessen wird.

\section{Integrierte, handlungsori- entierte Gesundheits- und Sozialberichterstattung (Berlin)}

In Erweiterung der seit den 1990er-Jahren vorgelegten „Sozialstrukturatlanten Berlin“ "wird in der Dissertation von Sabine Hermann [25] ein theoretisch entwickeltes indikatorengestütztes Berichterstattungssystem konzipiert und erprobt, 
das zahlreiche gesundheitliche und soziale Aspekte, wie Bildung, Erwerbsstatus und Einkommen, integriert.

Der Fokus der Arbeit liegt auf der Integration von Gesundheits- und Sozialdaten, um einen Beitrag zur Analyse regionaler gesundheitlicher und sozialer Ungleichheiten zu leisten. „Insgesamt enthält das Beobachtungssystem für acht Lebensbereiche - Demographie, Bildung, Einkommen, Erwerbsleben, Wohnen, Partizipation, Gesundheitszustand und Gesundheitsversorgung - 49 Indikatoren, davon 19 Leit- und 30 Ergänzungsindikatoren. Es basiert auf Daten der laufenden Raumbeobachtung für Deutschland, koordiniert und veröffentlicht vom Bundesamt für Bauwesen und Raumordnung (BBR)“ [25]. Die Berichtsbereiche decken sich im Wesentlichen mit denen, die im Projekt mit den 10 ostdeutschen GesundeStädte-Mitgliedern für die kommunale Ebene ausgewählt worden waren, wobei die Datenverfügbarkeit der einzelnen Indikatoren für Kommunen allerdings komplexer und schwieriger war.

Für Integration konstituierend ist die Vernetzung vormals getrennter Bereiche öffentlicher Planung und Berichterstattung auf der inhaltlichen, räumlichen, zeitlichen und Akteursebene. Die Arbeit fühlt sich dem New-PublicHealth-Ansatz verpflichtet, der die gesellschaftlichen Ursachen von Gesundheit/ Krankheit einbezieht bzw. versucht bei der Stärkung fördernder und Senkung hemmender gesundheitsrelevanter Faktoren (Determinanten der Gesundheit) anzusetzen. Auch die Nutzbarkeit des Ansatzes für integrierte Programme, wie das Gesunde-Städte-Netzwerk, Agenda 21, Gesundheit 21, Aktionsprogramm Umwelt und Gesundheit, wird erwähnt. Berichterstattung und Monitoring sollten in diesen Ansätzen eine Schlüsselstellung einnehmen, wobei dies aber nur sehr begrenzt realisiert wurde. Ebenso wie in der vorher beschriebenen kommunalen Berichterstattung spielt Nachhaltigkeit eine Rolle als Querschnittsthema, ergänzt durch ein weiteres Grundprinzip "Gender-Mainstreaming“, was als Alleinstellungsmerkmal des Ansatzes hervorgehoben wird [25].
Am Schluss werden Überlegungen zur Weiterentwicklung der Berliner Sozialberichterstattung dargestellt. Sie beziehen sich vor allem auf eine „zukünftige Organisationsstruktur, in deren Mittelpunkt eine Plattform für integrierte, partizipative Berichterstattung steht, als auch die stärkere Integration des Lebensbereichs Gesundheit unter das Dach der Sozialberichterstattung" [25].

$\mathrm{Zu}$ der Integration von Sozial- und Gesundheitsdaten gab es in Berlin Vorarbeiten seit etwa 1990, die u. a. in die Berliner Analyse zu „Sozialstruktur und Gesundheitszustand" und den beispielhaften "Sozialindikatorensatz" für die Sozialberichterstattung des Landes Brandenburg mündeten, der Gesundheitsindikatoren einschließt [26]. Weiterer wichtiger Meilenstein war der Berliner Sozialstrukturatlas von 2003 [27, 28].

Vermutlich unter Rückgriff auf die bahnbrechenden Vorarbeiten, für die als Schlüsselpersonen in der Berliner Verwaltung Meinlschmidt und Hermann verantwortlich waren (s.z. B. [29]), wurde in $₫ 5$ des Gesetzes über den öffentlichen Gesundheitsdienst (GDG) von 2006 die Aufgabe der „Integrierten Gesundheitsund Sozialberichterstattung" verankert. Sie soll eine Darstellung und Bewertung von Daten und Informationen, die für die Gesundheit und die soziale Lage der Bevölkerung bedeutsam sind, enthalten. Die Funktionen für Public Health werden ausführlich erläutert und weitreichende Verpflichtungen festgelegt, wie z.B.: „(2) Der öffentliche Gesundheitsdienst schreibt jährlich die Basisindikatoren fort und gewährleistet durch seine Informationstechnik Zugänglichkeit für die Adressaten der Berichterstattung " [30].

In der Folgezeit entstanden in Berlin tatsächlich viele Basis- und Spezialberichte [31], ergänzt durch weitere Elemente eines komplexen Informationssystems wie vor allem repräsentative Befragungen, genannt Gesundheitsbarometer [32] und das Gesundheits- und Sozialinformationssystem [33]. Als Kernstück, auch für die Rezeption in der Gesundheitsförderung, kann sicher der (nun allerdings schon einige Jahre zurückliegende) „Handlungsorientierte Sozialstrukturatlas Berlin 2013“ mit dem Untertitel „Grundlagen für passgenaue Sozialraum- planung sowie eine gesundheitsförderliche und soziale Stadtentwicklung" [34] gelten.

Eine umfassende Würdigung kann an dieser Stelle nicht geleistet werden. Interessant wäre aber die systematische Evaluation der Umsetzung des $\$ 5$ des Berliner GDG auf Landes- und Bezirksebene.

\section{Burgenland (Österreich) 2012 und 2017}

2012 verfasste die Fachhochschule Burgenland $\mathrm{GmbH}$ im Rahmen eines Forschungsprojekts und im Auftrag der burgenländischen Landesregierung den 3. Burgenländischen Gesundheitsbericht, explizit im Sinne eines umfassenden Public-Health-Reports [35]. Erstmalig wurde ein determinantenorientierter Bericht mit dem Fokus auf Prävention und Gesundheitsförderung (Schwerpunkt Kinder- und Jugendgesundheit) erstellt. Eingebunden waren politische Akteurinnen und Akteure aus den Bereichen Gesundheit, Umwelt und Soziales sowie Vertreterinnen und Vertreter von Krankenversorgungseinrichtungen, um die Akzeptanz des Berichtes zu erhöhen [36]. Der multimethodische Ansatz umfasste Sekundärdatenanalysen, eine standardisierte Befragung, leitfadengestützte Interviews und Fokusgruppen. Seit 2011 gibt es zusätzlich eine kontinuierliche Bürgerbefragung (Gesundheitsbarometer). Der Bericht berücksichtigte die verschiedenen Dimensionen der integrierten Gesundheitsberichterstattung entsprechend der Stufenleiter der Integration (• Abb. 1) und das Health-in-AllPolicies-Konzept und ließ auch schon unmittelbar nach Erscheinen praktische und politische Folgen erkennen [37].

Ein weiterer Bericht erschien 2017, ebenfalls erstellt von der Fachhochschule, Projektleitung: Barbara Szabo. Neben den Kapiteln Soziodemografie, Gesundheitszustand, Gesundheitsdeterminanten sowie „Gesundheitsförderungs-, Präventions- und Vorsorgeprojekte“ gibt es ein umfangreiches Kapitel "Gesundheits- und Krankenversorgung". Er ist der insgesamt vierte Bericht in etwa 5-jährlichen Abständen seit 2002 (und der zweite integrierte Bericht; [38]). 
Die Berichterstattung bezieht sich auf eine gesetzliche Grundlage [39], in der auch eine „Orientierung “ auf integrierte Versorgung, Health in All Policies und Public Health festgeschrieben ist. Von der Projektleiterin wurde mitgeteilt, dass weitere Integration in den zukünftig $\mathrm{zu}$ erwartenden Berichten angestrebt wird und dass auch in der Steiermark relativ gut integrierte Berichte erscheinen. Zusätzlich wurde auf die Datenbank aller österreichischen Gesundheitsberichte hingewiesen, die eine intensivere Recherche erlauben würde. Für diese Plattform haben sich die Bundesländer darauf geeinigt, Gesundheitsberichte in einem Abstand von etwa 5 Jahren zu aktualisieren und darüber hinaus auch nach Bedarf spezifische Themen aufzugreifen.

\section{Diskussion}

In der Diskussion sollen zunächst die methodischen Grenzen und Hindernisse der explorativen Recherche angesprochen werden. Auf der Basis einer Bilanz der gefundenen Projekte/Initiativen/ Ansätze wird abschließend auf die Barrieren und Chancen für integrierte (Gesundheits-)Berichterstattung eingegangen.

Als Hindernis für das Auffinden von integrierter Berichterstattung könnte die Beschränkung auf eine Internetrecherche angesehen werden. Es ist aber nicht sehr wahrscheinlich, dass deshalb solche Arbeiten übersehen wurden, da heute alles, was für eine Öffentlichkeit bestimmt ist, auch im Internet abgelegt wird. Außerdem erbrachte die E-MailBefragung der Schlüsselpersonen keine Hinweise auf nicht schon im Internet identifizierte Initiativen und Ansätze.

Problematischer sind die sehr eng gewählten Suchbegriffe (integrierte Gesundheitsberichterstattung und integrierte Berichterstattung). Im Rahmen der Recherche wurde deutlich, dass auch andere Begriffe verwendet werden, wie "Stadtgesundheitsprofil“ oder „Public-Health-Report“ (die allerdings bei zusätzlicher Überprüfung nicht zu weiteren Initiativen führten). Die Suche mit Begriffen wie Umwelt-, Nachhaltigkeits- und Sozialberichterstattung hätte möglicherweise $\mathrm{zu}$ Berichten geführt, in denen auch Gesundheit mehr oder weniger stark repräsentiert ist. Auch kann generell gesagt werden, dass in der Gesundheitsberichterstattung auf Bundesebene soziale Aspekte und in der Landesberichterstattung Umweltaspekte im Prinzip eine große Rolle spielen und sie insofern meist auch als zumindest bilateral integrierte Berichte zu bezeichnen wären. Ausgeklammert blieben auch integrierte Berichtssysteme auf höherer als Kommunal- und Landesebene, wie z. B. European Core Health Indicators (ECHI; [40]), oder kleinräumigere Ebenen, wie z. B. „Quartiersdiagnosen“ $[41,42]$. Insofern muss angenommen werden, dass - auch wenn wir die „prominentesten“ expliziten Ansätze gefunden haben - die Suchstrategie zu einem Underreporting solcher Berichterstattung geführt hat, die - ohne dies im Titel zu tragen - inhaltlich als „integriert“ zu bezeichnen wäre. Für NRW wurde dies in die Formulierung gefasst, dass sich „hinter vielen kommunalen Gesundheitsberichten schon längst integrierte Berichte verbergen" [43]. Eine Studie, die alle Berichte mit integrierten Anteilen, unabhängig von ihrer Betitelung, im Einzelnen analysiert, würde vermutlich $\mathrm{zu}$ anderen Schlussfolgerungen führen. Wie diese genau aussähen, lässt sich derzeit nicht sagen.

Bei aller Heterogenität der gefundenen Initiativen sind doch einige bilanzierende Feststellungen zur Beantwortung der Ausgangsfrage möglich, ob es im deutschsprachigen Bereich explizite Konzepte und eine öffentlich kommunizierte Praxis integrierter GBE gebe:

- Es gibt gut ausgearbeitete Indikatorensätze aus verschiedenen Quellen für die kommunale wie auch die Landesebene, die teilweise durch Primärerhebungen, wie z. B. ein „Gesundheitsbarometer“, ergänzt werden.

- Kernberichtsbereiche sind mit stark unterschiedlichen Akzentuierungen (neben Gesundheit und Demografie): Umwelt/Nachhaltigkeit, Soziales/ Armut und (überlappend) integrierte Stadtentwicklung. Sehr häufig sind Spezialberichte zum Thema Kinder und Jugendliche.

- Gesetzliche Vorgaben für die (integrierte) Berichterstattung und eine personelle Infrastruktur (Berlin, NRW) scheinen fördernde Faktoren für häufigere und inhaltlich vielfältigere Berichte $\mathrm{zu}$ sein.

- Wenn die normativen Vorgaben schwächer sind (Selbstverpflichtung im Rahmen von Forschung, „9Punkte-Papier“ des Gesunde-StädteNetzwerks), kommt es meist nur $\mathrm{zu}$ vereinzelter und nicht immer kontinuierlicher Berichterstattung.

- Es gibt einige Leuchtturmprojekte, wobei hohes Niveau der Integration und Kontinuität des Erscheinens nicht immer gemeinsam zu finden sind: Auf kommunaler Ebene sind das die Stadtgesundheitsprofile in Dresden, in NRW die Förderung von integrierter Berichterstattung durch die Landesbehörden und die Verbreitung integrierter Berichte auf kommunaler Ebene; Positivbeispiele auf Landesebene sind Berlin und das Burgenland.

- In den wissenschaftlichen Texten zu integrierter Berichterstattung und in den besten Praxisbeispielen wird als theoretische Fundierung explizit eine Public-Health-Strategie zugrunde gelegt, die sich auf Kernkonzepte wie die Determinanten der Gesundheit, den Public Health Action Cycle und den Settingansatz bezieht.

Positiver Befund ist also, dass es gute Theoriegrundlagen, gute Indikatorenkonzepte, positive gesetzliche Rahmenbedingungen wie in NRW, Berlin, das Burgenland sowie förderliche Faktoren wie Mitgliedschaft im Gesunde-StädteNetzwerk gibt. Daher ist erstaunlich, wie selten eine explizite integrierte Gesundheitsberichterstattung bisher geblieben ist. Dies lässt sich wohl vor allem verstehen, wenn man annimmt, dass die Hürden vielerorts zu hoch und die förderlichen Faktoren zu schwach sind.

$\mathrm{Zu}$ Hürden und Chancen der integrierten Berichterstattung gibt es zahlreiche Äußerungen.

$\mathrm{Zu}$ Hürden schreiben Boormann und Rosenkötter beispielsweise: „Bei allen Potenzialen kann jedoch nicht darüber hinweggesehen werden, dass es in der Praxis häufig Reibungsverluste in der Zusammenarbeit oder sogar eine generelle $\mathrm{Ab}$ - 
lehnung einer integrierten Herangehensweise gibt" [43]. Hermann stellt eingangs $\mathrm{zu}$ einem längeren Abschnitt [25] fest, dass trotz "positiver Resonanz" auf die Idee der integrierten Berichterstattung „die Praxis oft weit hinter den Erwartungen zurückbleibt“. Als Ursache wird die „Komplexität dieser Programme“ genannt, die „konzeptionelle und methodische Werkzeuge, die fachgerechte Analyse der aktuellen Situation, Prioritätensetzung, Entwicklung von Leitbildern und Zielen, Initiierung und Umsetzung von Maßnahmen sowie Prozess- und Ergebnisevaluation" erfordern [25]. Im Leitfaden aus NRW [23] werden als Hürden ausführlich beschrieben: fehlende finanzielle, personelle und fachliche Ressourcen, organisatorische und methodische Probleme sowie auf psychologischer Ebene Ängste und Vorbehalte. Auf der Basis systematischer Befragungen der 10 Modellstädte werden analoge Hürden weiter ausdifferenziert und daraus Schlussfolgerungen für ein „Zukunftsmodell für integrierte nachhaltigkeitsorientierte Berichterstattung“ entwickelt [44].

Chancen und Erfolgsfaktoren sind meist Schlussfolgerungen aus den gefundenen und teilweise überwundenen Hürden. Als notwendige Eckpunkte für integrierte Gesundheitsberichterstattung werden in dem österreichischen Ansatz gesehen: salutogenetisches Gesundheitsverständnis, indikatorengestützte Gesundheitsziele, Zusammenarbeit von Politik und Wissenschaft, gesetzliche Rahmenbedingungen sowie Ressourcen und Kapazitäten [45]. Im Pilotprojekt NRW mit 3 Kommunen [24] ergaben sich folgende notwendigen Voraussetzungen:

- politische Spitzen einbeziehen,

- fachübergreifende Zusammenarbeit sicherstellen,

- Führungsfunktion festlegen,

- institutionalisierte Strukturen schaffen,

- Zeit- und Personalbedarf sorgfältig planen,

- technische Machbarkeit vorab klären:

Wissensstand, Daten, Software.

Das erwähnte Zukunftsmodell [44] entstand vor allem aus den zahlreichen positiven Äußerungen und Ideen, die bei einer Kartenabfrage (Methode zur Ideen- findung) entstanden sind. Die 5 Kernforderungen (mit jeweils 8-10 Unterpunkten) lauteten:

\section{Berichterstattung aus der mit Freiwillig-} keit verbundenen Nachrangigkeit herausholen und in einen gesetzlichen Rahmen einfügen!

2. Infrastruktur für Datenpflege bzw. Datenfortschreibung nachhaltig sicherstellen! 3. Nachhaltige ämterübergreifende Kooperationsstrukturen schaffen!

4. Berichte für Bürger und Behörden schneller und billiger verfügbar machen durch neue Medien!

5. „Alles ist immer eine Zeit- und Ressourcen-Frage!?"

Für die weitere Entwicklung wäre es vermessen, einen „Königsweg“ im Sinne einer stark normierten generellen Anforderung für integrierte kommunale Berichterstattung vorzuschlagen. Im Sinne des Stufenmodells (• Abb. 1) kann Weiterentwicklung je nach dem bisher erreichten Stand stattfinden: Monothematische (Spezial-)Berichterstattung kann ergänzt werden durch Daten anderer Sektoren und Beteiligung der Akteure aus anderen Sektoren; bilaterale Fachberichte, wie es sie z. B. für den Bereich „Umwelt und Gesundheit“ gibt, können mit neuen Berichtsbereichen, etwa zu Sozialem, angereichert werden; im Idealfall werden arbeitsteilig organisierte „multilaterale“ Kooperationsstrukturen aufgebaut, die als Auftraggeber und Adressaten eine behörden- bzw. ämterübergreifende Instanz haben.

Positive Impulse für die Weiterentwicklung können auch aus der stark integriert angelegten Berichterstattung zu Nachhaltigkeitszielen (Sustainable Development Goals) kommen.

Die jüngst aktualisierte „Gute Praxis Berichterstattung“ [46] enthält an vielen Stellen Formulierungen, die als mehr oder weniger explizite Hinweise auf integrierte GBE verstanden werden können: Basis für ressortübergreifende Planung; inhaltliche Verflechtung von GBE mit unterschiedlichen $\mathrm{Be}$ richtssystemen, beispielsweise mit der Sozial-, Umwelt- oder Bildungsberichterstattung; Schnittmengen und Interdependenzen zwischen Gesundheits- und Sozialberichterstattung; Public-Health-
Relevanz als Grundlage für gesundheitspolitische Entscheidungen.

In Leitlinie 4 heißt es unter anderem: „Sie [die GBE] liefert Darstellungen und Analysen zu gesundheitlichen Determinanten, Rahmenbedingungen und anderen gesundheitsrelevanten Bereichen", in Empfehlung 4.3: „In die Berichterstattung werden die Erkenntnisse aus anderen Berichtssystemen wie etwa der Sozial-/Bildungs- und Umweltberichterstattung integriert", und schließlich in der Kriterienliste: „Bezogen auf die Fragestellung des Gesundheitsberichts erfolgt die Erstellung ... integrativ, d.h. in Zusammenarbeit mehrerer Fachbereiche/Ämter/Behörden“ [46].

Wenn die Empfehlungen der Guten Praxis Gesundheitsberichterstattung konsequent umgesetzt würden, wäre die Charakterisierung einer Gesundheitsberichterstattung als integriert eigentlich überflüssig: Ein guter Bericht wäre dann auch unweigerlich ein integrierter Bericht!

\section{Korrespondenzadresse}

Prof. Dr. Dr. Alf Trojan, MSc. (Lond.)

Institut für Medizinische Soziologie, Universitätsklinikum Hamburg-Eppendorf Martinistr. 52, 20251 Hamburg, Deutschland trojan@uke.de

Danksagung. Ich danke allen meinen Informantinnen und Informanten, insbesondere Frau Dr. Nicole Rosenkötter und Frau Ricarda Sahl sowie Dr. rer. soc. oec. Barbara Szabo, BA MA, die eine Vorfassung gelesen und mir Präzisierungen und Ergänzungen übermittelt haben. Außerdem danke ich den anonymen Gutachtern für zahlreiche Verbesserungsvorschläge!

Funding. Open Access funding provided by Projekt DEAL.

\section{Einhaltung ethischer Richtlinien}

Interessenkonflikt. A. Trojan gibt an, dass kein Interessenkonflikt besteht.

Für diesen Beitrag wurden von den Autoren keine Studien an Menschen oder Tieren durchgeführt Für die aufgeführten Studien gelten die jeweils dort angegebenen ethischen Richtlinien.

Open Access. Dieser Artikel wird unter der Creative Commons Namensnennung 4.0 International Lizenz veröffentlicht, welche die Nutzung, Vervielfältigung, Bearbeitung, Verbreitung und Wiedergabe in jeglichem Medium und Format erlaubt, sofern Sie den/die 
ursprünglichen Autor(en) und die Quelle ordnungsgemäß nennen, einen Link zur Creative Commons Lizenz beifügen und angeben, ob Änderungen vorgenommen wurden.

Die in diesem Artikel enthaltenen Bilder und sonstiges Drittmaterial unterliegen ebenfalls der genannten Creative Commons Lizenz, sofern sich aus der Abbildungslegende nichts anderes ergibt. Sofern das betreffende Material nicht unter der genannten Creative Commons Lizenz steht und die betreffende Handlung nicht nach gesetzlichen Vorschriften erlaubt ist, ist für die oben aufgeführten Weiterverwendungen des $\mathrm{Ma}$ terials die Einwilligung des jeweiligen Rechteinhabers einzuholen.

Weitere Details zur Lizenz entnehmen Sie bitte der Lizenzinformation aufhttp://creativecommons.org/ licenses/by/4.0/deed.de.

\section{Literatur}

1. Thiele W, Trojan A (Hrsg) (1990) Lokale Gesundheitsberichterstattung. Hilfen auf dem Weg zu einer neuen Gesundheitspolitik? Asgard, St. Augustin, $\mathrm{S} 11-221$

2. Fehr R, Fertmann $R$ (2018) StadtGesundheit in Hamburg und Altona. In: Fehr R, Trojan A (Hrsg) Nachhaltige StadtGesundheit Hamburg. OekomVerlag, München, $\mathrm{S32}-41$

3. Reincke JJ, Medicinal-Collegium (Hrsg) (1901) Die Gesundheitsverhältnisse Hamburgs im neunzehnten Jahrhundert. S4 (zit. n. 1, S. 20)

4. Osius N, Trojan A, Flesch-Janys D, Berger J, Kappos A D (2001) Indikatoren für eine gesunde und nachhaltige Stadtentwicklung. Projektbericht, unveröffentlicht

5. Ziese T, Trojan A (2015) Gesundheitsberichterstattung. In: BZgA (Hrsg) Leitbegriffe der Gesundheitsförderung https://doi.org/10.17623/ BZGA:224-i028-1.0

6. Osius N, Trojan A, Kappos AD, Flesch-Janys D (2000) Indikatoren für eine gesunde und nachhaltige Stadtentwicklung. Dokumentationsmappe zum Workshop am 16. Nov. 2000 in Hamburg, unveröffentlicht

7. Süß W, Möller H, Trojan A, Fehr R (Hrsg) (2004) Integrierte Basis-Berichterstattung für gesündere Städte und Kommunen. Quellen, Auswahlprozess und Profile für einen Indikatorensatz. Wissenschaftliche Reihe, Bd. 17. Landesinstitut für den Öffentlichen Gesundheitsdienst NRW (lögd), Bielefeld (komprimiert in Süß W, Glismann W, Trojan A (2005) Integrierte nachhaltigkeitsorientierte Berichterstattung - Kernindikatoren für Kommunen und Städte. Gesundheitswesen 67:150-154)

8. Süß W (2009) Integrierte Berichterstattung für integrierte Handlungskonzepte auf kommunaler Ebene - konzeptionelle und normative Anforderungen. In: Kuhn J, Böcken J (Hrsg) Verwaltete Gesundheit: Konzepte der Gesundheitsberichterstattung in der Diskussion. Mabuse, Saarbrücken, S183-199

9. Süß W, Schäfer I, Trojan A (Hrsg) (2007) Integrierte (Gesundheits-) Berichte. Konzeptionelle Überlegungen und Umsetzungserfahrungen. Shaker, Aachen

10. StadtHalle(Saale), FachbereichPlanen (61)(o.J.)Integriertes StadtentwicklungskonzeptHalle. http:// www.halle.de/de/Verwaltung/Online-Angebote/ Veroeffentlichungen/?RecID=757. Zugegriffen: 17. Febr. 2020 (S261-289)
11. Stadt Gera (2014) Integrierte Sozialberichterstattung. https://www.gera.de/sixcms/detail. php?id=21730\&_nav_id $1=146704 \&$ nav id2 $=146712 \&$ lang=de. Zugegriffen: 17. Febr. 2020

12. Stadt Gera (2018) SOZIALPLAN 2018- 2023 - Integriertes Handlungskonzept der StadtGera. https:// www.gera.de/sixcms/detail.php?id=21730\& nav_id $1=146704 \&$ nav_id $2=146712 \&$ \&lang $=$ de Zugegriffen: 17. Febr. 2020

13. Voigt I (2016) Integrierte Sozialplanung, Gesundheitsberichterstattung und zielgruppenorientier te chancengleiche Gesundheitsförderung in der Stadt Gera/Thüringen. Masterarbeit

14. Stadtverwaltung Gera (o.J.) Netzwerk Gesunde Kommune Gera. https://www.gera.de/ sixcms/detail.php?id=196292\&_nav_id1 $=$ \& nav_id $2=\&$ _lang $=$ de. Zugegriffen: 17 . Febr. 2020

15. JorzikM (2020) briefl (Mitteilung vom 17.02.2020)

16. Integrierte Gesundheitsberichterstattung Weimar (2017) Projekt: Kommunale Präventionskette. https://stadt.weimar.de/stadtverwaltung/ projekte/kommunale-praeventionskette/. Zugegriffen: 17. Febr. 2020

17. Thüringer Ministerium für Soziales, Familie und Gesundheit (2013) Tagung des Thüringer Ministeriums. https://www.gesundheitlichechancengleichheit.de/veranstaltungen/termine/ integrierte-berichterstattung-wege-undperspektiven-zur-staerkung-von-kommunen/. Zugegriffen: 17. Febr. 2020

18. Landeshauptstadt Dresden (2016) Stadtgesundheitsprofile Dresden. https://www.dresden.de/ de/leben/gesundheit/who/gesundheitsprofile. php.Zugegriffen: 17. Febr. 2020 (S.4)

19. Bearingpoint/FOGS im Auftrag des MfJFG des Landes NRW (2003) Evaluation des Gesetzes über den öffentlichen Gesundheitsdienst ÖGDG des Landes NRW. Entwurf des Schlußberichts, S182-191

20. Landeszentrum Gesundheit Nordrhein-Westfalen (LZG.NRW) (o.J.) Datenbank (freiwillig eingestellter) kommunaler Gesundheitsberichte NRW. https://www.lzg.nrw.de/ges_bericht/ komm_gbe/Datenbank_Berichte/index.html.Zu gegriffen: 17. Febr. 2020

21. Rosenkötter N, Borrmann B (2014) Einblicke in die kommunale und kleinräumige Gesundheitsberichterstattung - die Datenbank kommunale Gesundheitsberichterstattung in Nordrhein-Westfalen. Gesundheitswesen 76:A151

22. Rosenkötter N, Borrmann B (2015) Entwicklungsstand einer integrierten kommunalen Gesundheitsberichterstattung in Nordrhein-Westfalen. Gesundheitswesen 16:V50. https://doi.org/10. 1055/s-0035-1546890

23. Hamm R, Kreutzer F (2018) Pilotprojekt für eine integrierte kommunale Umwelt-, gesundheitsund Sozialberichterstattung. (Auftraggeber Ministerium für Umwelt, Landwirtschaft, Natur und Verbraucherschutz des Landes NRW), S 4 ff. https://www.umwelt-und-gesundheit.nrw.de/ fileadmin/redaktion/PDF-Dateien/Pilotprojekt_ integrierte_Berichterstattung.pdf. Zugegriffen: 17. Febr. 2020 (https://www.umwelt.nrw.de/ umwelt/umwelt-und-gesundheit/masterplanumwelt-und-gesundheit/)

24. MAGS NRW, MULNV NRW (2019) Integrierte Berichterstattung und Planung - Erfolgsfaktoren, Hürden und gute Argumente. https://www. umwelt-und-gesundheit.nrw.de/fileadmin/ redaktion/PDF-Dateien/Veroeffentlichung_ Integrierte_Berichterstattung_und_Planung. pdf. Zugegriffen: 17. Febr. 2020 (http://www. sozialberichte.nrw.de/sozialberichterstattung nrw/Veranstaltungen/index.php)

25. Hermann S (2006) Konzept einer integrierten, handlungsorientierten Gesundheits- und Sozialberichterstattung im regionalen Ansatz. Theorien, Methoden, Anwendungsfelder. Dissertation,Technische Universität Berlin, S.105306

26. Bardehle D, Blettner M, Laaser U (2001) Gesundheits- und soziodemographische (sozialepidemiologische) Indikatoren in der Gesundheitsund Sozialberichterstattung. Bundesgesundheitsblatt Gesundheitsforschung Gesundheitsschutz 44:382-393. https://doi.org/10.1007/ s001030050456

27. (2003) Berliner Gesundheits-und Strukturatlas. https://www.google.com/search? client=firefox-b- $d \& q=$ berliner+Gesundheits+und+Strukturatlas+2003. Zugegriffen: 17. Febr. 2020

28. Landes-Gesundheitsbericht Hamburg (2001) Stadtdiagnose 2. Zweiter Gesundheitsbericht für Hamburg. http://www.kinderumweltgesundheit. de/index2/pdf/gbe/6049_1.pdf. Zugegriffen: 17. Febr. 2020

29. Meinlschmidt G, Hermann S (2007) Integrierte Gesundheits- und Sozialberichterstattung. In: Reintjes R, Klein S (Hrsg) Gesundheitsberichterstattung und Surveillance. Messen, Entscheiden und Handeln. Huber, Bern, S 114-123

30. Senatsverwaltung für Justiz, Verbraucherschutz und Antidiskriminierung (o.J.) ÖGD-Gesetz Berlin. http://gesetze.berlin.de/jportal/?quelle=jlink\& query $=\%$ C3\%96GesDG+BE\&psml=bsbeprod. psml\&max=true\&aiz=true. Zugegriffen: 17 . Febr. 2020

31. Senatsverwaltung für Gesundheit, Pflege und Gleichstellung, der Patientenbeauftragten, der Landesdrogenbeauftragten und dem Landesbeauftragten für Psychiatrie (o.J.) Basisberichte Berlin mit unterschiedlichen Schwerpunkten. https://www.berlin.de/sen/gesundheit/service/ gesundheitsberichterstattung/veroeffentlichun gen/basisberichte/.Zugegriffen:17. Febr. 2020

32. Gesundheitsbarometer Berlin (Verantwortlich Dr. S. Hermann) (o.J.) Gesundheitsbarometer Berlin (Verantwortlich Dr. S. Hermann). https://www.berlin.de/sen/gesundheit/service/ gesundheitsberichterstattung/veroeffentlichun gen/gesundheitsbarometer/. Zugegriffen: 17. Febr. 2020

33. Gesundheits- und Sozialinformationssystem Berlin (2020) Gesundheits- und Sozialinformationssystem Berlin. http://www.gsi-berlin.info/gsi_ aktuell.asp.Zugegriffen: 17. Febr. 2020

34. Gesundheit Berlin-Brandenburg e.V. (2013) Handlungsorientierter Sozialstrukturatlas Berlin. https://www.gesundheitlichechancengleichheit.de/handlungsorientiertersozialstrukturatlas-berlin-2013/.Zugegriffen: 17. Febr. 2020

35. Burgenland (2012) Burgenländischer Gesundheitsbericht, Berichtszeitraum 2017 - 2012. https://goeg.at/sites/goeg.at/files/2017-06/bgb_ 2012_langfassung_0.pdf. Zugegriffen: 17. Febr. 2020

36. Schnabel F, Szabo B, Gollner E (2014) Beitrag in: Tagungsband 8 . Forschungsforum der österreichischen Fachhochschulen. http://hdl.handle.net/20. 500.11790/189. Zugegriffen: 17. Febr. 2020

37. Szabo B, Schnabel F, Gollner E (2014) Integrierte Gesundheitsberichterstattung als konzeptionelle 
Grundlage für intersektorale Zusammenarbeit. Prävention 36(1):18-21

38. Szabo B et al (2018) Burgenländischer Gesundheitsbericht 2017 Kurzfassung. Erstes Burgenländisches Rechenzentrum, Eisenstadt (Amt der Burgenländischen Landesregierung - Abteilung 6 Soziales und Gesundheit (Hrsg))

39. (o.J.) Vereinbarung Artikel 15a Bundesverfassungsgesetz Österreich. https://www.ris.bka. gv.at/GeltendeFassung.wxe?Abfrage=LrVbg\& Gesetzesnummer=20001137. Zugegriffen: 17. Febr. 2020

40. European Core Health Indicators. https://ec. europa.eu/health/indicators/echi/list_en. Zugegriffen: 17. Febr. 2020

41. Wolf K, Süß W, Lorentz C (2019) Kleinräumige Gesundheitsberichterstattung für die Situationsanalyse und -beobachtung: Ansätze, Methoden und Datenquellen. In: Nickel S, Lorentz C, Süß W, Wolf K, Trojan A (Hrsg) Qualitätsentwicklung und Verstetigung quartiersbezogener Gesundheitsförderung. Lit, Münster, S138-149

42. Mossakowski K, Nickel S, Schäfer I, Süß W, Trojan A Werner S (2007) Die Quartiersdiagnose: Daten und Ansätze für ein stadtteilorientiertes Präventionsprogramm des Öffentlichen Gesundheitsdienstes - erste Ergebnisse eines Forschungsprojektes. Präv Gesundheitsf 2(1):82-89

43. Borrmann B, Rosenkötter N (2016) Integrierte Berichterstattung für ressortübergreifende kommunale Gesundheitspolitik. Public Health Forum 24(4):272-274

44. Trojan A, Süß W (2007) Hürden und Chancen handlungsorientierter integrierter Berichterstattung. Von der Problemanalyse zum Zukunftsmodell. In: Süß W, Schäfer I, Trojan A (Hrsg) ntegrierte (Gesundheits-) Berichte. Konzeptionelle Überlegungen und Umsetzungserfahrungen. Shaker, Aachen, S261-289

45. Ochsenhofer D (2012) Der Weg vom Gesundheitsbericht zum Public Health Bericht in Österreich. Diplomarbeit. https://www.diplomarbeiten24. de/document/307775.Zugegriffen: 17. Febr. 2020

46. Starke D, Tempel G, Butler J, Starker A, Zühlke C, Borrmann B (2019) Gute Praxis Gesundheitsberichterstattung - Leitlinien und Empfehlungen 2.0. J Health Monit 5(S1):1-22. https://doi.org/10. 25646/6058 